\title{
La auto-evaluación como elemento de medición para el aseguramiento de la calidad en las Instituciones Tecnológicas. ${ }^{1}$
}

\section{Self Evaluation as a Measurement Element for Assuring of Quality in Technological Institutions.}

Jorge Augusto Valencia Valencia ${ }^{2}$, Sandra Milena Tamayo Aristizábal ${ }^{3}$. Fundación Universitaria María Cano y Corporación Escuela Tecnológica, Colombia.

Artículo recibido en el mes de mayo de 2013; artículo aceptado en el mes de julio de 2013.

Citación del artículo: Valencia, J. \& Tamayo, S. (2013). La auto-evaluación como elemento de medición para el aseguramiento de la calidad en las Instituciones Tecnológicas. I+D Revista de Investigaciones, 1(1), 75-81.

\section{Resumen}

El objetivo del presente estudio es describir algunos factores asociados a la reducida participación de las Instituciones Técnicas y Tecnológicas (ITT) afiliadas a la Asociación Colombiana de Instituciones de Educación Superior con Formación Técnica Profesional y/o Tecnológica (ACIET) en el proceso de Acreditación de sus programas ante el Sistema Nacional de Acreditación (SNA). Se sigue un método descriptivo y se recogen datos de 33 instituciones. a partir de lo cual se ofrece un panorama sobre este problema educativo.

Palabras clave: Acreditación, autoevaluación, calidad, educación superior, formación técnica y tecnológica.

\section{Abstract}

The main goal of this research is to describe some factors related to the limited participation of the Technical and Technological Institutions (ITT) associated to the Colombian Association of Higher Education with Technical, Technological or Proffessional Training (ACIET) in the accreditation process of their academic programs facing the National Accreditation System (SNA). A descriptive method is followed and data from 33 institutions is gathered, presenting a perspective of this educational problem.

Keywords: Accreditation, self evaluation, quality, higher education, technical and technological training.

\footnotetext{
'Artículo de investigación científica, de enfoque cuantitativo, resultado parcial de un proyecto de investigación en desarrollo, denominado: La auto-evaluación como elemento de medición para el aseguramiento de la calidad en las Instituciones Tecnológicas, desarrollado en el grupo de investigación de la ACIET. Calle 9 No. 23-55, PBX: 6352525.
} 


\section{Introducción}

Desde el año de 1992 se expidió la Ley 30 de Educación Superior en conexión con la Constitución Política de Colombia de 1991, la cual considera, en sus artículos 53, 54 y 55, la creación del Sistema de Aseguramiento para la Calidad de la Educación Superior (SACES) como referente voluntario a adoptar, para el cumplimiento de altos requisitos de calidad educativa de los programas académicos profesionales; y la Ley 749 de 2002, para los técnicos y tecnológicos en las instituciones educativas públicas y privadas del país, colocando a las Instituciones Técnicas y Tecnológicas (ITT) al mismo nivel respecto del proceso de Acreditación de sus programas. La conformación del Consejo Nacional de Acreditación (CNA) y finalmente la Autoevaluación Institucional, fueron, por un lado, el organismo de control de la misma, y por el otro, la tarea permanente de mejoramiento del proceso educativo que se planteó como mecanismo conducente a la garantía de la calidad educativa en Colombia.

La Ley 1188 de 2009 reglamentada por el decreto 1295 de 2010 para los Registros Calificados, exige un enfoque de calidad de manera obligatoria para todas las Instituciones de Educación Superior en Colombia. Así mismo, el CNA determinó los elementos sobre los cuales se sustenta la Certificación y Acreditación en Colombia, para lo cual se deben desarrollar las 40 características y 10 factores en el proceso de autoevaluación. Según el MEN, de los 10.415 programas, de ITT existentes afiliadas a la Asociación Colombiana de Instituciones de Educación Superior con Formación Técnica
Profesional y/o Tecnológica (ACIET) en todo el país, sólo el 13\% están acreditadas. Son en total 71 , de las cuales sólo tres tienen acreditación institucional; de igual forma entre ellas suman en total 1.400 programas y sólo 21 están acreditados.

La elección de las ITT del país, de someterse al proceso de Acreditación de forma voluntaria, ha sido muy reducida, pese a que se reconoce la necesidad de acogerse a un sistema de evaluación para servir a sus objetivos educacionales e innovación. En virtud de ello, la ACIET junto con la participación del Ministerio de Educación Nacional (MEN) y el CNA, han buscado incrementar la participación, aunque a la fecha no se visualizan los resultados de la motivación constante que se ha desarrollado desde la Asociación hacia las instituciones tecnológicas y técnicas del país, de las que sólo un $2 \%$ se hallan acreditadas. A partir del anterior panorama se buscó mediante el presente estudio descriptivo, explorar algunas variables asociadas con la no participación de las instituciones de educación técnica y tecnológica en los procesos de acreditación de sus programas.

\section{Método}

\section{Tipo de estudio.}

Se siguió un tipo de investigación descriptiva transversal y se utiliza la triangulación de información para poder nutrir las descripciones del fenómeno estudiado.

\section{Participantes.}

Se toma como población a las 61 ITT afiliadas a la ACIET, de las cuales respondieron a la 
encuesta así: Públicas 5 y Privadas 28, por tipo de Institución, que ofertan programas técnicos profesionales y tecnológicos, y se consideraron: Instituciones Técnicas Profesionales 10, Tecnológicas 12, Instituciones Universitarias 9, Fundaciones Universitarias 3 y Universidades 3. Es de anotar, que la legislación colombiana permite la redefinición de las instituciones, lo que ha llevado a las ITT a que se redefinieran a Institución Universitaria, ofertando programas profesionales; así mismo, las Instituciones Universitarias pueden ofertar programas Técnicos Profesionales y Tecnológicos, lo que no permite identificar solamente instituciones con este carácter.

\section{Materiales e instrumentos. encuesta.}

La encuesta fue validada por pares académicos, un instrumento que indaga por el desarrollo del proceso de autoevaluación, aplicada a las 33 instituciones de formación técnica y tecnológica afiliadas a ACIET; indaga sobre sus procesos de calidad aplicada a la gestión académica y administrativa.

\section{Procedimiento.}

Se llevó a cabo la estructuración de las dimensiones de evaluación a partir de las cuales se formularon las preguntas constitutivas de la encuesta. El repertorio de preguntas fue analizado y depurado por un equipo de jueces, al cual se sometió para su validación.

Una vez estructurada y evaluada la encuesta, se contactó a las ITT de la ACIET para definir los mecanismos de aplicación y de retorno de los instrumentos diligenciados.
Se realizó la aplicación de la encuesta en cada ITT participante y se recogieron los formatos de aquellas que los retornaron de forma completa y en los plazos estipulados.

Se llevó a cabo el levantamiento de la base de datos en la cual se registraron las respuestas a cada uno de las preguntas realizadas.

Se procesaron los datos en un paquete estadístico para obtener los parámetros descriptivos, y ofrecer los resultados de las encuestas aplicadas.

\section{Resultados}

Se presentan algunos resultados de la encuesta de 15 preguntas aplicadas a las ITT participantes y que permite el análisis del problema presentado para la investigación.

La figura 1, muestra que las instituciones técnicas o tecnológicas que se hallan acreditadas ante el CNA, son sólo dos de las 33 encuestadas, lo que equivale a un $6 \%$ de ellas.

Figura 1. Instituciones técnicas o tecnológicas acreditadas ante CNA.



Fuente: Autor.

La figura 2, muestra los resultados obtenidos con respecto a la pregunta sobre el conocimiento 
que tienen las ITT, del procedimiento que deben seguir si aspiran a tener reconocimiento de alta calidad de sus programas académicos. Al respecto se observa que todas las Instituciones conocen el procedimiento para realizar el proceso, lo cual denota que han existido los mecanismos de información para la participación de las instituciones en este proceso.

Figura 2. Conocimiento de procedimiento de acreditación de programas por las ITT.

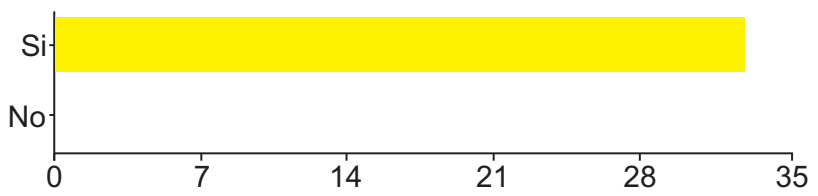

Si $33 \quad 100 \%$

No $\quad 0 \quad 0 \%$

Fuente:Autor.

Para la participación en un proceso de acreditación, es muy importante la motivación; por lo tanto, se indagó acerca de qué motiva a las ITT a participar de este proceso, destacándose respuestas como el mejoramiento continuo, la posibilidad de autorregularse y el reconocimiento público de que es una Institución de Calidad, como se observa en la tabla 1.

Tabla1. Frecuencias y porcentajes por motivo percibido por las ITT para entrar a la Acreditación

\begin{tabular}{lrr}
\hline Criterios Motivacionales & f & $\%$ \\
\hline Organización Académica & 8 & $13 \%$ \\
Reconocimiento público de la Calidad & 12 & $20 \%$ \\
Posibilidad de autoevaluarse y autorregularse & 14 & $23 \%$ \\
Mejora continua & 25 & $42 \%$ \\
Otra & 1 & $2 \%$ \\
\hline
\end{tabular}

Fuente. Autor.
En la tabla 2, se observan las frecuencias y porcentajes de cada beneficio percibido por las ITT de la condición de estar acreditado como programa ante el CNA. Los beneficios percibidos que más se destacan son el fortalecimiento de la comunidad académica, la modernización académica y las posibilidades de la internacionalización.

Tabla 2. Frecuencias y porcentajes de beneficios percibidos por las ITT de estar acreditados.

\begin{tabular}{lcc}
\hline Beneficios percibidos de la acreditación & $\mathrm{f}$ & $\%$ \\
\hline $\begin{array}{l}\text { Modernización académica e } \\
\text { internacionalización }\end{array}$ & 21 & $31 \%$ \\
$\begin{array}{l}\text { Fortalecimiento de la } \\
\text { comunidad académica }\end{array}$ & 23 & $34 \%$ \\
$\begin{array}{l}\text { Participación en redes y } \\
\text { asociaciones }\end{array}$ & 11 & $16 \%$ \\
$\begin{array}{l}\text { Acuerdos internacionales } \\
\text { Otra }\end{array}$ & 9 & $13 \%$ \\
\hline
\end{tabular}

Fuente: Autor.

En la figura 3, se muestra el número de ITT que tienen implementados sistemas de certificación de calidad diferentes al del CNA. Se destaca que el $56 \%$ de las Instituciones participantes poseen certificado de calidad, lo que contrasta con el $94 \%$ que no participan en los procesos de Acreditación; ésto denota el compromiso de las Instituciones por evaluarse y documentar sus procesos para lograr el mejoramiento continuo, independientemente de las agencias certificadoras.

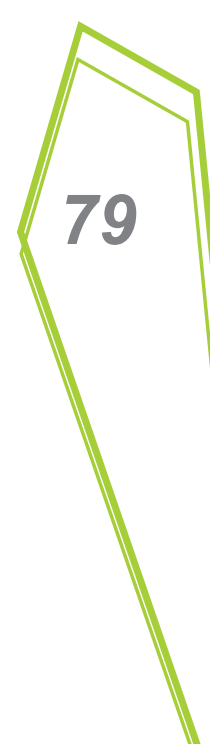


Figura 3. Sistemas de certificación de la calidad en las ITT diferentes al del CNA.

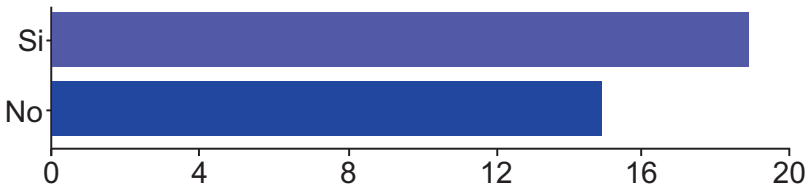

Si $19 \quad 56 \%$

No $15 \quad 44 \%$

Fuente: Autor.

La tabla 3, muestra las agencias certificadoras de procesos de calidad diferentes al CNA, al que se acogen algunas de las ITT evaluadas.

Tabla 3. Frecuencias y porcentajes de beneficios percibidos por las ITT de estar acreditados.

\begin{tabular}{lcc}
\hline Beneficios percibidos de la acreditación & $\mathrm{f}$ & $\%$ \\
\hline ICONTEC & 13 & $68 \%$ \\
SGM & 2 & $11 \%$ \\
BUREAU VERITAS & 2 & $11 \%$ \\
Otra & 2 & $11 \%$
\end{tabular}

Fuente. Autor.

Se reconoce la participación en este proceso a través de agencias como el Instituto colombiano de normas técnicas ICONTEC y otras organizaciones que se ajustan a los requerimientos internacionales; de igual forma, es de considerar, que este proceso de certificación no riñe con el proceso de acreditación, los cuales pueden ser complementarios.

Con respecto a la pregunta sobre la dificultad que han encontrado como ITT durante el proceso de autoevaluación, los resultados mostraron que para las Instituciones participantes, las dificultades principales son las siguientes: (a) la extensión de las encuestas, (b) el cuestionamiento sobre asuntos de los cuales no se está suficientemente informado, (c) la escasa motivación para responder las encuestas en el caso de egresados y empresarios.

\section{Discusión}

Es importante considerar que de las 93 ITT del país, sólo 10 se encuentran inscritas en el SACES, primer requisito para participar del proceso de Acreditación, lo que corresponde al $10,8 \%$; es de recordar que en la ACIET, se encuentran afiliadas 79 Instituciones que ofertan programas técnicos profesionales, tecnológicos y profesionales; para esta investigación participaron 33 Instituciones respondiendo a la encuesta, en conjunto ofrecen novecientos cuarenta y seis (946) programas y se encuentran acreditados setenta y nueve (79) programas en Alta Calidad por el CNA, lo que representa un $8 \%$ del número de programas que ofertan las Instituciones participantes en el proceso. De las 33 ITT encuestadas, sólo un $6 \%$ se encuentran acreditadas ante el CNA, lo que en números, es sólo dos instituciones; esto significa que es ínfimo y poco representativo el número de ellas que han acogido las directrices Estatales al respecto y por lo tanto que no se encuentran bajo un régimen que controle sus procesos de calidad hacia la excelencia, al menos desde el parámetro de medición pública del país. Esta situación, sin embargo, no parece deberse al desconocimiento de la existencia del proceso de acreditación de los programas técnicos y tecnológicos, puesto que en la pregunta número dos, las 33 instituciones reportan conocer este mecanismo de control de la calidad hacia estándares 
competitivos. Adicionalmente puede evidenciarse que más de la mitad de las instituciones poseen certificado de calidad con otras agencias de acreditación, lo que denota un compromiso de las Instituciones por evaluarse y documentar sus procesos para lograr el mejoramiento continuo, a pesar de que no estén sujetas a los previstos por agencias del Estado.

Dentro de las motivaciones para hacer parte de este mecanismo de calidad estatal, las respuestas encontradas muestran que las principales están relacionadas con la mejora de los estándares educativos, lo cual permite destacar que para las instituciones encuestadas, el interés por adherir a un mecanismo de autoevaluación como el previsto por el Estado, permite hallarse en sintonía con parámetros de excelencia y calidad formativa.

En cuanto a los beneficios que se perciben de la acreditación, se valora la actualización que se llega a alcanzar y las posibilidades de cumplir con estándares que facilitan procesos de globalización, en los cuales se abren los horizontes de actuación y las perspectivas educativas y de proyección educativas tanto para las instituciones como para los estudiantes y docentes.

Con respecto a la pregunta sobre la dificultad que han encontrado como ITT durante el proceso de autoevaluación, los resultados muestran que los instrumentos y procesos con los cuales se indaga, pueden tener dificultades de diverso tipo que hacen que la labor de reporte de la información pueda resultar largo, tedioso y que genere efectos motivacionales en quienes deben responderlos, que limiten su alcance.

\section{Referencias}

Consejo Nacional de Acreditación (CNA) (2013). Autoevaluación con fines de acreditación de programas. Bogotá. Colombia. Recuperado de: http://www.cna.gov.co/1741/articles186376_guia_autoev_2013.pdf

Departamento Nacional de Planeación (2011). Plan Nacional de Desarrollo 2010- 2014. Bogotá, Colombia. Recuperado de: https://www.dnp.gov.co/LinkClick.aspx?filetic ket=4-J9V-FE2pI\%3D\&tabid $=1238$

Ministerio de Educación Nacional (2011). Plan estratégico del sector educativo 2011- 2014. Bogotá. Colombia. Recuperado de: http://www.mineducacion.gov.co/cvn/1665/arti

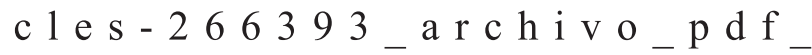
mariafernandacampo.pdf

Ospina, A. ( 2004). Régimen jurídico de la educación en Colombia. Régimen general y especial de la educación superior de Colombia. Bogotá: Leyer.

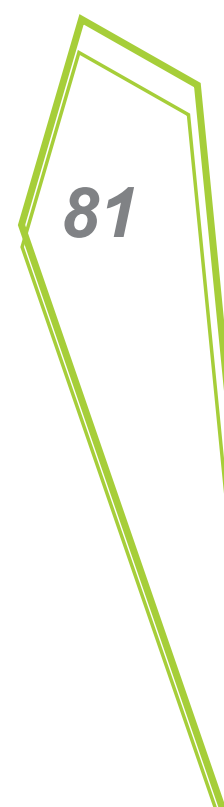

